\title{
CONTINUITY PROPERTIES OF SAMPLE FUNCTIONS OF MARKOV PROCESSES $\left({ }^{(}\right)$
}

BY

\section{J. R. KINNEY}

1. Introduction. We shall be concerned with the continuity properties of sample functions of Markov processes.

We let $\Omega=\{\omega\}$ be a space in which a completely additive probability measure, $P(\Lambda)$, is defined over a Borel field $\mathcal{F}_{\omega}=\{\Lambda\}$ of subsets of $\Omega$. A random variable is any function measurable with respect to $\mathcal{F}_{\omega}$. A stochastic process is any family of random variables $\left\{x_{t}(\omega), t \in T^{0}\right\}$, where $T^{0}$ is a linear set. A sample function is the function $x_{t}(\omega)$, considered as a function of $t$, for fixed $\omega$. We shall sometimes say "almost all sample functions," "almost all $\omega$," "with probability one," or "almost everywhere" (written a.e.), meaning for all $\omega$ except an $\omega$-set of measure zero.

A regularity condition known as separability (applied to processes) will often be used. $A$ separable process $\left\{x_{t}(\omega), t \in T^{0}\right\}$ is a process for which there is a sequence $t_{i}$ of parameter values, dense in $T^{0}$, and a set $\Lambda, P(\Lambda)=0$, such that, for $\omega \notin \Lambda$,

$$
\inf _{t \in I T^{0}} x_{t}(\omega)=\inf _{t_{i} \in I T^{0}} x_{t_{i}}(\omega), \quad \sup _{t \in I T^{0}} x_{t}(\omega)=\sup _{t_{i} \in I T^{0}} x_{t_{i}}(\omega)
$$

for every interval $I$. We shall also use a somewhat stronger condition which we shall denote as property $\mathrm{S}^{*}$. A stochastic process $\left\{x_{t}(\omega), t \in T^{0}\right\}$ will be said to have property $\mathrm{S}^{*}$ if there is a sequence $R=t_{i}$ of parameter values dense in $T^{0}$ and a set $\Lambda, P(\Lambda)=0$, such that for $\omega \notin \Lambda$, for $B$ any closed set, I any open $t$-interval, if $x_{t}(\omega) \in B, t \in I R$, then $x_{t}(\omega) \in B, t \in I T^{0}$.

We shall suppose given a function $P(X)$, a completely additive probability measure defined for $X \in \mathcal{F}$, the field of linear Borel sets, and a function $P(t, x ; T, X)$, defined for $0 \leqq t<T \leqq T^{\prime}$, for all real $x, X \in \mathcal{F}$, such that:

(a) $P(t, x ; T, X)$ for fixed $t, T, X$ is a Baire function of $x$,

(b) $P(t, x ; T, X)$ for fixed $t, x, T$ is a completely additive probability measure defined over $\mathcal{F}$.

(c) For $0 \leqq t<s<T \leqq T^{\prime}$, all $x, X \in \mathcal{F}$,

$$
P(t, x ; T, X)=\int_{-\infty}^{\infty} P(t, x ; s, d y) P(s, y ; T, X) .
$$

Received by the editors May 5, 1951 and, in revised form, May 6, 1952.

(1) This paper is a revision of a thesis submitted to the University of Illinois under the direction of J. L. Doob, to whom the author owes many suggestions and comments. The paper was written while the author was engaged on a research project directed by Professor William Feller, at Cornell University, for the Office of Naval Research. 
It will be convenient to define $P(t, x ; t, X)$ to be 1 for $x \in X, 0$ for $x \notin X$. With this convention (a), (b), and (c) with $t \leqq s \leqq T$ are still satisfied. The equation (1) is known as the Chapman-Kolmogoroff equation.

A function $P(t, x ; T, X)$ satisfying (a), (b), and (c) will be called a transition probability function.

It has been shown [Doob, 3 ] that under these conditions there is a Markov process $\left\{x_{t}(\omega), 0 \leqq t \leqq T^{\prime}\right\}$ for which

$$
\begin{gathered}
P\left(x_{0}(\omega) \in X\right)=p(X), \\
P\left(x_{T}(\omega) \in X \mid x_{t}(\omega)=x\right)=P(t, x ; T, X),
\end{gathered}
$$

$A$ Markov process is a stochastic process $\left\{x_{t}(\omega), t \in T^{0}\right\}$ such that, for $t_{1}<t_{2}<\cdots<t_{m-1}<t_{m}, t_{i} \in T^{0}, X$ a Borel set,

$P\left(x_{t_{m}}(\omega) \in x \mid x_{t_{i}}(\omega)=x_{i}, i=1, \cdots, m-1\right)=P\left(x_{t_{m}}(\omega) \in X \mid x_{t_{m-1}}(\omega)=x_{m-1}\right)$, a.e.

The probabilities $P\left(x_{T}(\omega) \in X \mid x_{t}(\omega)=x\right)$ are known as the transition probabilities.

This approach is somewhat less general than to suppose a Markov process to be given and to attempt to determine a transition probability function from it which satisfies the Chapman-Kolmogoroff equation. However, the assumption of property $S^{*}$ will be no restriction on the transition probability functions, since, by a theorem of Doob [1, Chapter II, Theorem 2.4], to any stochastic process $\left\{y_{t}(\omega), t \in T^{0}\right\}$ there corresponds a stochastic process $\left\{\tilde{y}_{t}(\omega), t \in T^{0}\right\}$, separable, or with property $S^{*}$, such that $P\left(\tilde{y}_{t}(\omega)=y_{t}(\omega)\right)=1$, for every value of $t$.

$A$ martingale process $\left\{y_{t}(\omega), t \in T^{0}\right\}$ is a stochastic process with $E\left\{\left|y_{t}(\omega)\right|\right\}$ $<\infty, t \in T^{0}$, such that:

(i) for each $t \in T^{0}$, there exists a Borel field, $\mathcal{F}_{t}, \mathcal{F}_{t} \subset \mathcal{F}_{\omega}$;

(ii) $y_{t}(\omega)$ is equal, for almost all $\omega$, to a function measurable with respect to $\mathcal{F}_{t}$;

(iii) for $t_{1}<t_{2}, \mathcal{F}_{t_{1}} \subset \mathcal{F}_{t_{2}}$, and

$$
E\left\{y_{t_{2}}(\omega) \mid \mathcal{F}_{t_{1}}\right\}=y_{t_{1}}(\omega),
$$

a.e.

One of the properties of $P(t, x ; T, X)$ which we shall use is that the Markov property implies that the process $\left\{P\left(t, x_{t}(\omega) ; T, X\right), t<T, t \in T^{0}\right\}$, considering $P\left(t, x_{t}(\omega) ; T, X\right)$ for fixed $X, T, t$ as a random variable, $t$ as the parameter of the family, is a martingale process. In fact, for $s<t$,

$$
P\left(t, x_{t}(\omega) ; T, X\right)=E\left\{P\left(s, x_{s}(\omega) ; T, X\right) \mid \mathcal{F}_{t}\right\},
$$

where $\mathcal{F}_{t}$ is the Borel field of $\omega$-sets generated by conditions of the form $x_{u_{i}}(\omega)$ $\leqq a_{i}, u_{i}<t, i=1,2, \cdots, n$.

As an example of a process of this sort, we consider the transition probability functions of a Brownian motion process. A Brownian motion process is a separable process such that:

(i) for each $t, s, x_{t+8}(\omega)-x_{s}(\omega)$ has a Gaussian distribution with mean zero, 
variance $\alpha|t|$,

(ii) for $t_{1}<t_{2}<\cdots<t_{n}$,

$$
x_{t_{2}}(\omega)-x_{t_{1}}(\omega), \cdots, x_{t_{n}}(\omega)-x_{t_{n-1}}(\omega)
$$

are independent random variables. Wiener showed in 1923 that almost all sample functions of this process are continuous. If $x_{0}(\omega)$ is independent of the increments $x_{t+\varepsilon}(\omega)-x_{s}(\omega)$, for all positive $s$ and $t$, then the process, considered for positive $t$, is a Markov process. A function satisfying our assumptions (a), (b), and (c), $\alpha=1$, is

$$
P(t, x ; T, X)=\int_{X} \frac{e^{-(y-x)^{2} / 2(T-t)}}{(2 \pi(T-t))^{1 / 2}} d y .
$$

There is also a corresponding density function,

$$
p(t, x ; T, y)=\frac{e^{-(y-x)^{2} / 2(T-t)}}{(2 \pi(T-t))^{1 / 2}} .
$$

If $x_{t}(\omega)$ is the random variable of the Brownian motion process, then

$$
P\left(t, x_{t}(\omega) ; T, X\right)=\int_{X} \frac{e^{-\left(y-x_{t}(\omega)\right)^{2} / 2(T-t)}}{(2 \pi(T-t))^{1 / 2}} d y
$$

is the random variable of the martingale process $\left\{P\left(t, x_{t}(\omega) ; T, X\right), 0 \leqq t \leqq T\right\}$. The random variable

$$
p\left(t, x_{t}(\omega) ; T, y\right)=\frac{e^{-\left(y-x_{t}(\omega)\right)^{2} / 2(T-t)}}{(2 \pi(T-t))^{1 / 2}}
$$

is also the random variable of a martingale process.

In $\$ 2$ we use the fact that $\left\{P\left(t, x_{t}(\omega) ; T, X\right), t<T, t \in T^{0}\right\}$ is for each $T, X$, a martingale process by applying a theorem of Doob [1, Chapter VII, Theorem 11.5]:

THEOREM. Except possibly for a set of sample functions of probability zero, the sample functions of a separable martingale process $\left\{y_{t}(\omega), t \in T^{0}\right\}$ have the following properties:

(i) they are bounded on every $t$-set of the form $\{t<s\} \cap T^{0}, s \in T^{0}$,

(ii) they have finite left-(right-)hand limits at every $t \in T^{0}$ which is a limit point of $T^{0}$ from the left (right).

This application shows that Markov processes with property $\mathrm{S}^{*}$ whose transition probability functions satisfy certain regularity conditions have sample functions which are almost all continuous except for strict jumps. A function $f(t)$ is continuous except for jumps if $\lim _{t \uparrow t_{0}} f(t)=f\left(t_{0}^{-}\right)$and $\lim _{t \downarrow t_{0}} f(t)=f\left(t_{0}^{+}\right)$exist and are finite and either $f\left(t_{0}^{-}\right) \leqq f\left(t_{0}\right) \leqq f\left(t_{0}^{+}\right)$or $f\left(t_{0}^{-}\right) \geqq f\left(t_{0}\right)$ $\geqq f\left(t_{0}^{+}\right)$for all $t_{0}$. A function is continuous except for strict jumps if always 
$f\left(t_{0}^{+}\right)=f\left(t_{0}\right)$ or $f\left(t_{0}^{-}\right)=f\left(t_{0}\right)$.

In $\$ 3$ we show that if the transition probabilities of the Markov process satisfy an additional condition of the form:

$$
P\left(\left|x_{t+h}(\omega)-x_{t}(\omega)\right|>\epsilon \mid x_{t}(\omega)=x\right)=o(h) h \rightarrow 0, \quad \text { a.e., }
$$

the sample functions of the Markov process are almost all continuous.

In $\$ 4$ we give examples illustrating the results.

Interest in Markov process has centered around two main types: the first has been defined by assumptions on the functions

$$
E\left\{x_{t+h}(\omega)-x_{t}(\omega) \mid x_{t}(\omega)=x\right\}
$$

and

$$
E\left\{\left(x_{t+h}(\omega)-x_{t}(\omega)\right)^{2} \mid x_{t}(\omega)=x\right\}
$$

of the sort:

$$
\begin{aligned}
E\left\{x_{t+h}(\omega)-x_{t}(\omega) \mid x_{t}(\omega)\right. & =x\}=h m(t, x)+o(h), \\
E\left\{\left(x_{t+h}(\omega)-x_{t}(\omega)\right)^{2} \mid x_{t}(\omega)=x\right\} & =h \sigma^{2}(t, x)+o(h),
\end{aligned}
$$

where restrictions are made on the regularity of $m(t, x), \sigma(t, x)$ and the rapidity with which $o(h) \rightarrow 0$ as $h \rightarrow 0$. The second type has been defined by the condition $\lim _{h \rightarrow 0} P\left(x_{t+h}(\omega)=x_{t}(\omega)\right)=1$. The second type of process is sometimes called purely discontinuous.

Kolmogoroff [1] showed that the transition probability functions of processes of both types satisfied certain differential equations. For the first type these differential equations were two second order partial differential equations of parabolic type, known as the Fokker-Planck equation and its adjoint, closely associated with diffusion equations. In the case of the second type, under certain restrictions on the state space, he showed that they satisfy two systems of linear differential equations. He alse showed that for more complicated types of processes, they satisfied a certain integro-differential equation. Feller $[1,2]$ investigated the converse problem for a class of processes of the first type, the second type, and a mixture of the two. Under certain assumptions he found that there do exist Markov processes whose transition probability functions satisfy these differential equations.

Fortet [1], in an investigation of processes of the first type, showed that the sample functions of a certain class of processes whose transition probability functions satisfy the Fokker-Planck equation are almost all continuous. Doeblin [1] investigated the second type of Markov process. He found sufficient conditions that the sample functions be continuous except at a finite number of values of $t$, in finite closed intervals, and constant on the intervals between successive pairs of these values. He also showed the transition probability functions to satisfy certain integral equations. For the first type of process he announced conditions that $x_{t}(\omega) /\left(1+\left|x_{t}(\omega)\right|\right)$ be continuous [3]. 
Ito [1] has succeeded in establishing a relation between a large class of processes of the first type and the Brownian motion by means of a stochastic integral. He exhibited a large class of processes of mixed type as solutions of a certain stochastic integral equation. Processes of the second type were further investigated by Doeblin [2], Feller [3], and Doob [4, 5]. Bernstein [1] considered a class of processes of the first type, by investigating processes given by considering the transition probabilities on a net in the parameter interval. He showed the limit of the approximating transition probability functions to satisfy the Fokker-Planck equations. He also constructed examples to show the type of discontinuity which could occur in the sample functions of the process if the conditions were violated. Doob [5] investigated processes of the second type in a space with a countable number of points. He showed that if certain systems of differential equations, the generalizations of the systems derived by Kolmogoroff, do not have a unique set of solutions, the sample functions may have discontinuities more complicated than jumps.

2. Conditions under which the sample functions have right- and left-hand limits. We suppose throughout the discussion $\left\{x_{t}(\omega), 0 \leqq t \leqq T^{\prime}\right\}$ to be a Markov process with property $\mathrm{S}^{*}$ with an initial distribution $p(X)$ and transition probability functions $P(t, x ; T, X)$ defined for all $x, t, T, 0 \leqq t \leqq T$ $\leqq T^{\prime}, X \in \mathcal{F}, \mathcal{F}$ the field of the Borel sets, satisfying the conditions described in the introduction. We have seen that the Markov property implies that $\left\{P\left(t, x_{t}(\omega) ; T, X\right), 0 \leqq t \leqq T\right\}$ is a martingale process. It is known that almost all sample functions of separable martingale processes have finite right- and left-hand limits at every $t, 0 \leqq t \leqq T$. We wish to investigate properties of the $x_{t}(\omega)$ process which can be deduced from this property and certain conditions imposed on $P(t, x ; T, I)$ where $I$ is an open interval. These conditions are as follows:

C: We let $I(x, a)=(x-a, x+a)$. Then the condition $\mathrm{C}$ is that

$$
P(t, x ; t+h, I(x, a))=1+f(h), f(h) \rightarrow 0 \quad \text { as } h \rightarrow 0 .
$$

We write $\mathrm{C}(,,$, ) where the first argument will denote restriction on $t$, the second argument will denote restrictions on $x$, the third argument will denote restrictions on $a$, and the fourth will specify the manner in which $f(h) \rightarrow 0$ as $h \rightarrow 0$. The restrictions on $\mathrm{C}$ governed by the behavior of $t$ will be: " $f$," that $\mathrm{C}$ hold for all fixed $t$; or " $u$," that $\mathrm{C}$ hold uniformly in $t$. The restrictions on $\mathrm{C}$ governed by the behavior of $x$ will be: " $f$," that $\mathrm{C}$ hold for all fixed $x$; " $b$," that $\mathrm{C}$ hold, uniformly for all $x$ in any bounded interval; " $u$," that $\mathrm{C}$ hold, uniformly in $x$. The restrictions on $\mathrm{C}$ governed by the behavior of a will be: "w," that $\mathrm{C}$ hold for some $a(x)$ such that $x-a(x) \rightarrow \infty$, as $x \rightarrow \infty, x+a(x) \rightarrow-\infty$ as $x \rightarrow-\infty$; " $a$," that $\mathrm{C}$ hold for some fixed $a$; " $\epsilon$, " that $\mathrm{C}$ hold for every $a>0$. The restrictions on $\mathrm{C}$ governed by the behavior of $f(h)$ will be: " $o(1)$, " that $f(h) \rightarrow 0$ as $h \rightarrow 0$; "o(h)," that $f(h) / h \rightarrow 0$ as $h \rightarrow 0$. 
Thus, for example, the condition $\mathrm{C}(u, u, a, o(1))$ signifies that

$$
P(t, x ; t+h, I(x, a)) \rightarrow 1 \quad \text { as } h \rightarrow 0
$$

uniformly in $t$, uniformly in $x$, for some fixed $a$. The condition $\mathrm{C}(u, b, \epsilon, o(h))$ signifies that

$$
[P(t, x, t+h, I(x, a))-1] / h \rightarrow 0 \quad \text { as } \quad h \rightarrow 0
$$

uniformly, in $t$, uniformly in $x$ in any bounded interval, for any $a>0$. "C" is used to denote a continuity condition.

$\mathrm{D}$ : The condition $\mathrm{D}$ is that

$$
P(t, x ; T, I) \rightarrow \delta(x, I) \quad \text { as } \quad T-t \downarrow 0
$$

for $x$ not an end point of $I$, where $\delta(x, I)=1$ for $x$ interior to $I, \delta(x, I)=0$ for $x$ interior to the complement of $I$. We shall write $\mathrm{D}($, , , ) where the first argument will denote restrictions on $t$, the second, restrictions on $x$, the third, restrictions on $T$, the fourth, restrictions on $I$. The restrictions on $\mathrm{D}$ governed by the behavior of $t$ are: " $f$, , D is to hold for all fixed $t$; " $u, " \mathrm{D}$ is to hold uniformly in $t$. Restrictions governed by the behavior of $T$ will be similarly indicated. It will be noted that the restrictions governed by $t$ and those governed by $T$ are not independent. That is, if $\mathrm{D}$ holds uniformly in $T$, then $\mathrm{D}$ holds uniformly in $t$, and vice versa. The restrictions on $\mathrm{D}$ governed by the behavior of $x$ are: " $b$," that $\mathrm{D}$ hold uniformly in $x$ for $x$ in any bounded set $A$, bounded away from the end points of $I$; " $u$," that $\mathrm{D}$ hold uniformly in every set $A$, bounded or not, bounded away from the end points of $I$; finally, if $\mathrm{D}$ is to hold for every bounded interval $I$, we denote the restriction by " $b$ "; if $\mathrm{D}$ is to hold for every interval $I$, bounded or not, we denote the restriction by " $u$."

Thus, for example, $\mathrm{D}(f, b, b)$ means that

$$
P(t, x ; T, I) \rightarrow \delta(x, I) \quad \text { as } \quad T-t \downarrow 0
$$

for all fixed $t$, uniformly for $x$ in any bounded set $A$ at a positive distance from the end points of $I$, for every bounded interval $I . \mathrm{D}(u, u, u)$ means that

$$
P(t, x ; T, I) \rightarrow \delta(x, I) \quad \text { as } \quad T-t \downarrow 0
$$

uniformly in $t$, uniformly for $x$ in any set bounded away from the end points of $I$, for any interval $I$, bounded or not. The " $\mathrm{D}$ " is to denote a boundary condition of $\delta$-function type.

$\mathrm{R}(\infty)(\mathrm{R}(-\infty))$ : The condition $\mathrm{R}(\infty)(\mathrm{R}(-\infty))$ is that

$$
P(t, x ; T, I) \rightarrow 0 \quad \text { as } x \rightarrow \infty \quad(x \rightarrow-\infty)
$$

for all bounded intervals $I$, for $T-t$ sufficiently small, independently of $I$. " $\mathrm{R}$ " is a condition on the "return."

We introduce the following tables, where the conditions given in the arguments of $\mathrm{C}$ and $\mathrm{D}$ may be conveniently found: 
C: $P(t, x ; t+h, I(x, a))=1+f(h)$, to hold, subject to restrictions

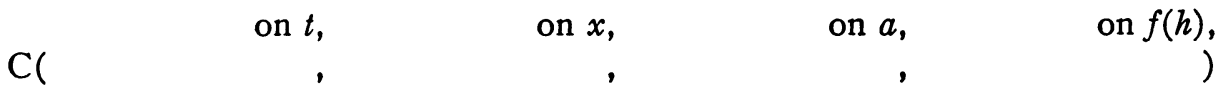

$f:$ for each fixed $t \quad f$ : for each fixed $w:$ for $I(x, a) \quad o(1): f(h) \rightarrow 0$ as

$x$ $=(x-a(x)$, $h \rightarrow 0$ $x+a(x))$

$u$ : uniformly in $t \quad b$ : uniformly for where

$$
\begin{array}{llr}
x \in A, \text { any } & |(x \pm a(x))| \rightarrow \infty & \text { as } h \rightarrow 0 \\
\text { bounded set } & \text { as } x \rightarrow \infty &
\end{array}
$$

$u$ : uniformly in $a$ : for some fixed

$x$

$a$

$\epsilon$ : for all $a>0$

D: $P(t, x ; T, I) \rightarrow \delta(x, I)$, as $T-t \downarrow 0$, to hold subject to restrictions

$\mathrm{D}($

on $t, \quad$ on $x, \quad$ on $T$,

on $I$,

- conditions on $T f$ : for each $x$ not - conditions on - for each $I$ specified

an end point $t$ specified

of $I$

$f$ : for each fixed $t \quad b$ : uniformly for $f$ : for each fixed $b$ : for all bounded
$x$ in any
bounded set
bounded away
from the end
points of $I$

$T$ intervals $I$

$u$ : uniformly in $t \quad u$ : uniformly for $u$ : uniformly in $u$ : for all inter$x$ in any set $T$

bounded away

vals $I$ bound-

from the end

ed or not

points of $I$

Conditions $\mathrm{C}$ and $\mathrm{D}$ are not independent. $\mathrm{C}(u, b, \epsilon, o(1))$ is equivalent to $\mathrm{D}(u, b, b)$. We first show that $\mathrm{C}(u, b, \epsilon, o(1))$ implies $\mathrm{D}(u, b, b)$. We choose $A$ to be any bounded set bounded away from the end points of $I$, for instance $A \subset(-M, M)$, the distance from $A$ to the end points of $I$ to be greater than $\epsilon$. Then, since $\mathrm{C}(u, b, \epsilon, o(1))$ implies that $P(t, x ; t+h, I(x, \epsilon))$ $=o(1)_{(h \rightarrow 0)}$ uniformly for $x$ in $(-M, M)$, and since $I(x, \epsilon) \subset I$, if $x \in A \cap I$, we have, for $x \in A \cap I, 1 \geqq P(t, s ; t+h, I) \geqq P(t, x ; t+h, I(x, \epsilon))=1-o(1)_{(h \rightarrow 0)}$, for $x \in A \cap c I$, since $c I(x, \epsilon) \supset I, 0 \leqq P(t, x ; t+h, I) \leqq 1-P(t, x ; t+h, I(x, \epsilon))$ $=o(1)_{(h \rightarrow 0)}$. Since the $o(1)$ by assumption $\mathrm{C}(u, b, \epsilon, o(1))$ is uniform for 
$x \in(-M, M)$, the approach of $P(t, x ; t+h, I)$ to $\delta(x, I)$ is uniform in $x$, for $x \in A$. We next show that $\mathrm{D}(u, b,, b)$ implies $\mathrm{C}(u, b, \epsilon, o(1))$. We suppose this to be false. Then there exists a bounded sequence $x_{i}$ and a sequence $r_{i} \rightarrow r_{0}$ such that, for some $\epsilon>0, P\left(r_{i}, x_{i} ; r_{0}, I(x, \epsilon)\right)>\delta$. Since the $x_{i}$ are bounded, we may choose a subsequence $x_{i_{j}}$ approaching a limit, $\xi$. We pick $I=(\xi-2 \epsilon, \xi+2 \epsilon)$. Then for $j$ sufficiently large, $\left(x_{i_{j}}-\epsilon, x_{i_{j}}+\epsilon\right) \subset(\xi-2 \epsilon$, $\xi+2 \epsilon)$ so

$$
P\left(r_{i_{j}}, x_{i_{j}} ; r_{0}, I\right)>P\left(r_{i_{j}}, x_{i_{j}} ; r_{0}, I\left(x_{i_{j}}, \epsilon\right)\right)>\delta .
$$

This contradicts $\mathrm{D}(u, b, \quad, b)$. Hence the assertion is true.

$\mathrm{C}(u, u, \epsilon, o(1))$ implies $\mathrm{D}(u, u, \quad, u)$. This can be shown by the same argument as that used above.

$\mathrm{C}(u, b, \epsilon, o(1))$ and $\mathrm{C}(u, u, w, o(1))$ imply $\mathrm{D}(u, u,, b)$. To prove this statement we show that for any fixed $I$, any $\epsilon>0,|P(t, x ; T, I)-\delta(x, I)|<\epsilon$ for $x$ in a set bounded away from the end points of $I, T-t$ sufficiently small. We take $M$ so large that for $x>M,|x \pm a(x)|>\max _{y \in I}(|y|+1)$. We delete $\epsilon$-neighborhoods of the end points of $I$ from $(-M, M)$ to obtain $A$. Then if $x \in I \cap A$ or if $x \in A \cap c I$, the proof that $\mathrm{C}(u, b, \epsilon, o(1))$ implies $\mathrm{D}(u, b, \quad, b)$ shows that, for $T-t$ small enough, $|P(t, x ; T, I)-\delta(x, I)|<\epsilon$. For $x \notin A, x \geqq M, \mathrm{C}(u, u, w, o(1))$ implies that, for $T-t$ small enough, $P(t, x ; T, I)<\epsilon$. This establishes the implication.

It can be shown that $\mathrm{D}(f, f,, b)$ is equivalent to $\mathrm{C}(f, f, \boldsymbol{\epsilon}, \boldsymbol{o}(1))$.

Theorem I. (i) $\mathrm{D}(f, f,, b)$ implies that $p \lim _{t \downarrow t_{0}} x_{t}(\omega)=x_{t_{0}}(\omega)$ for all $t_{0}$.

(ii) $\mathrm{D}(\quad, f, f, b)$ implies that $p \lim _{t \uparrow t_{0}} x(\omega)=x_{t_{0}}(\omega)$ for all $t_{0}$.

Proof of (i): $\mathrm{D}(f, f,, b)$ implies

$$
\lim _{h \downarrow 0} P\left(\left|x_{t+h}(\omega)-x_{t}(\omega)\right|>\epsilon\right)=\lim _{h \downarrow 0} P\left(\left|x_{t+h}(\omega)-x_{t}(\omega)\right|>\epsilon \mid x_{t}(\omega)\right) d P=0
$$

since the integrand is bounded by 1 and approaches 0 . The same argument is valid for the proof of (ii).

We shall have frequent occasion to take limits of $x_{t}(\omega)$, for fixed $\omega$, through the countable dense set in $0 \leqq t \leqq T^{\prime}$ used to define the property $\mathrm{S}^{*}$. We shall call this set $R$. Henceforth $r, r^{0}, r^{\prime}, r_{i}$, and $r_{i}^{\prime}$ will be values from this set. Since for all $\omega$ not in the exceptional set $\Lambda$ of the property $S^{*}$,

$$
\limsup _{r \uparrow t(r \downarrow t)} x_{r}(\omega)=\limsup _{s \uparrow t,(s \downarrow t)} x_{s}(\omega), \quad \liminf _{r \uparrow t(r \downarrow t)} x_{r}(\omega)=\liminf _{s \uparrow t,(s \downarrow t)} x_{s}(\omega),
$$

for all $t$, when right- and left-hand limits of $x_{r}(\omega)$ exist, so do those of $x_{t}(\omega)$, and the two coincide, with probability one. Since for $\omega$ not contained in $\Lambda$, the exceptional set of property $S^{*}$,

$$
\lim _{n \rightarrow \infty} \underset{|r-t|>1 / n}{G . L . B .} x_{r}(\omega) \leqq x_{t}(\omega) \leqq \lim _{n \rightarrow \infty} \underset{|r-t|>1 / n}{\text { L.U.B. }} x_{r}(\omega),
$$


for all $t \notin R$, the only sample function discontinuities which can occur for values of $t$ not in $R$, when right- and left-hand limits exist, are jumps, with probability one. If $x_{r}(\omega)$ has right- and left-hand limits $x_{t_{0}^{+}}^{+}(\omega)$ and $x_{t_{0}}^{-}(\omega)$ at $t_{0}$, property $\mathrm{S}^{*}$ implies that $x_{t_{0}}(\omega)$ lies in $B$, where

$$
\begin{aligned}
B= & \left\{x \mid x_{t_{0}}-(\omega)-\epsilon \leqq x \leqq x_{t_{0}}-(\omega)+\epsilon\right\} \\
& \bigcup\left\{x \mid x_{t_{0}}+(\omega)-\epsilon \leqq x \leqq x_{t_{0}}+(\omega)+\epsilon\right\}
\end{aligned}
$$

for every $\epsilon$. This implies that $x_{t_{0}}(\omega)=x_{t_{0}}(\omega)$ or $x_{t_{0}}(\omega)=x_{t_{0}^{+}}^{+}(\omega)$, for $t_{0} \notin R$, with probability one.

By a theorem of Doob [1, Chapter II, Theorem 2.4] to any stochastic process, $\left\{y_{t}(\omega), t \in T^{0}\right\}$, there corresponds a separable stochastic process $\left\{\tilde{y}_{t}(\omega), t \in T^{0}\right\}$, such that at each $t, y_{t}(\omega)=\tilde{y}_{t}(\omega)$, with probability one. The exceptional set may vary with $t$. For each $t=r \in R$, we eliminate the exceptional set for each of the following martingale processes: $\left\{P\left(t, x_{t}(\omega) ; t_{0}, I\right)\right.$, $\left.0 \leqq t \leqq t_{0}\right\}$ where $I$ has rational end points, $t$ is the parameter of the process, and ranges over $\left(0, t_{0}\right), 0 \leqq t_{0} \leqq T_{0}^{\prime}$. There are a countable number of such processes. The remaining set we call $\Omega_{t_{0}}^{\prime}$. Since we have eliminated only a countable number of sets of zero measure, $P\left(\Omega_{t_{0}}^{\prime}\right)=1$. Each of the equivalent separable processes is a martingale process since each coincides with a martingale process for each $t$, except for a set of measure zero. We call the equivalent separable martingale processes $\left\{\widetilde{P}\left(t, x_{t}(\omega) ; t_{0}, I\right), 0 \leqq t \leqq t_{0}\right\}$. Using the theorem of Doob cited in the introduction, each has sample functions having finite right- and left-hand limits for each $t, 0 \leqq t \leqq t_{0}$, with probability one. We eliminate the exceptional set for each of the countable number of equivalent separable martingale processes from $\Omega_{t_{0}}^{\prime}$ obtaining $\Omega_{t_{0}}$. Since only a countable number of sets of measure zero are eliminated, $P\left(\Omega_{t_{0}}\right)=1$. We shall later use $\Omega^{*}=\bigcap_{0<r<r^{\prime}} \Omega_{r}$. Since for each $r, P\left(\Omega_{r}\right)=1$, it follows that $P\left(\Omega^{*}\right)=1$.

TheOREM II. (i) $\mathrm{D}(, b, f, b)$ implies that at any $t_{0}$, for almost all $\omega$,

$$
\lim _{t \uparrow t_{0}} \frac{x_{t}(\omega)-x_{t_{0}}(\omega)}{1+x_{t}^{2}(\omega)}=0 .
$$

(ii) $\mathrm{D}(, u, f, b)$ implies that at any $t_{0}$, for almost all $\omega$,

$$
\lim _{\imath \uparrow t_{0}} x_{t}(\omega)=x_{t_{0}}(\omega) .
$$

(The exceptional set depends on $t_{0}$, in each case.)

Proof of (i). We suppose an $\omega \in \Omega_{t_{0}}$ such that there are $r_{i}, r_{i} \uparrow t_{0}, r_{i}^{\prime}, r_{i}^{\prime} \uparrow t_{0}$, for which $x_{r_{i}}(\omega) \rightarrow a, a \neq \pm \infty, x_{r_{i}}(\omega) \rightarrow b, b \neq \pm \infty, a \neq b$. We choose $I$ to be an open interval with rational end points, at a positive distance from $b$, with $a$ in its interior. The $A$ of condition $\mathrm{D}$ we choose to be a closed interval interior to $I$ containing $a$ in its interior, plus a bounded closed interval with $b$ 
in its interior at a positive distance from $I$. The assumption $\mathrm{D}(, b, f, b)$ implies $P\left(r_{i}, x_{r_{i}}(\omega) ; t_{0}, I\right) \rightarrow 1$ as $i \rightarrow \infty, P\left(r_{i}^{\prime}, x_{r_{i}}(\omega) ; t_{0}, I\right) \rightarrow 0$ as $i \rightarrow \infty$. This implies that $\tilde{P}\left(t, x_{t}(\omega) ; t_{0}, I\right)$ has an oscillatory discontinuity at $t_{0}$, contrary to the construction of $\Omega_{t_{0}}$. Hence $x_{r}(\omega)$ has at most one finite limiting value from the left at $t_{0}$. Since $\left\{x_{t}(\omega), 0 \leqq t \leqq T^{\prime}\right\}$ has property $\mathrm{S}^{*}, x_{t}(\omega)$ has at most one finite limiting value from the left at $t_{0}$. By Theorem I(ii), $x_{t_{0}}(\omega)$ is a limiting value from the left at $t_{0}$, with probability one. This establishes (i).

Proof of (ii). By (i), with probability one, for $\omega \in \Omega_{t_{0}}$, there exist $r_{i}$, such that $x_{r_{i}}(\omega) \rightarrow x_{t_{0}}(\omega)$. From the above, the only other possible limiting values are $\infty,-\infty$. We assume that there exist $r_{i}^{\prime}, r_{i}^{\prime} \uparrow t_{0}$ such that $\left|x_{r_{i}}(\omega)\right| \rightarrow \infty$. We choose $I$ as any open interval with rational end points containing $x_{t_{0}}(\omega) . A$ we choose to be the real line, deleted by neighborhoods of the end points of $I$ at a positive distance from $x_{t_{0}}(\omega)$. Then $\mathrm{D}(, u, f, b)$ implies that

$$
P\left(r_{i}, x_{r_{i}}(\omega) ; t_{0}, I\right) \rightarrow 1 \text { as } i \rightarrow \infty ; \quad P\left(r_{i}^{\prime}, x_{r_{i^{\prime}}}(\omega) ; t_{0}, I\right) \rightarrow 0 \text { as } i \rightarrow \infty .
$$

This implies that $P\left(t, x_{t}(\omega) ; t_{0}, I\right)$ has an oscillatory discontinuity at $t_{0}$, contrary to the construction of $\Omega_{t_{0}}$. Hence the supposition $\left|x_{r_{i}}(\omega)\right| \rightarrow \infty$ is false. This establishes (ii).

TheOREM III. (i) $\mathrm{D}(u, b, \quad, b)$ implies that at any $t_{0}$, for almost all $\omega$,

$$
\lim _{t \downarrow t_{0}} \frac{x_{t}(\omega)-x_{t_{0}}(\omega)}{1+x_{i}^{2}(\omega)}=0 .
$$

(ii) $\mathrm{D}(u, u,, u)$ implies that at any $t_{0}$, for almost all $\omega$,

$$
\lim _{t \downarrow t_{n}} x_{t}(\omega)=x_{t_{0}}(\omega) .
$$

Proof of (i). As in the proof of Theorem II (i), (i) will be true if $x_{r}(\omega)$ can have at most one finite limiting value as $r \downarrow t_{0}$ for all $\omega \in \Omega^{*}$. We assume that there is an $\omega \in \Omega^{*}$ for which there exist sequences $r_{i} \downarrow t_{0}, r_{i}^{\prime} \downarrow t_{0}$, such that $x_{r_{i}}(\omega) \rightarrow a, a \neq \pm \infty, x_{r_{i}}^{\prime}(\omega) \rightarrow b, b \neq \pm \infty, a \neq b$. We choose $I, A$, as in the proof of Theorem II (i). We choose $r^{\prime}>t_{0}$, so near $t_{0}$ that, for $t \in\left(t_{0}, r^{\prime}\right)$,

$$
P\left(t, x ; r^{\prime}, I\right)>0.9 \text {, if } x \in A \cap I, \quad P\left(t, x ; r^{\prime}, I\right)<0.1 \text {, if } x \in A \cap c I \text {. }
$$

This choice of $r^{\prime}$ is possible under the assumption $\mathrm{D}(u, b,, b)$ because of the uniformity which is assumed in $t$. Choosing $i$ so large that for $j>i$, $r_{j}<r^{\prime}, r_{j}^{\prime}<r^{\prime}, x_{r_{j}}(\omega) \in I \cap A, x_{r_{j}^{\prime}}^{\prime}(\omega) \in c I \cap A$, we have

$$
P\left(r_{j}, x_{r_{j}}(\omega) ; r^{\prime}, I\right)>0.9, \quad P\left(r_{j}^{\prime}, x_{r^{\prime}}(\omega) ; r^{\prime}, I\right)<0.1,
$$

for $j>i$. This implies an oscillatory discontinuity of $\tilde{P}\left(t, x_{t}(\omega) ; r^{\prime}, I\right)$ contrary to the construction of $\Omega^{*}$. Hence $x_{r}(\omega)$ can have but one finite limit from the right. The same argument as in the proof of Theorem II (i) shows that this implies that $x_{t}(\omega)$ can have but one finite limiting value from the 
right, and that, with probability one, this limiting value is $x_{t_{0}}(\omega)$.

The same modification of the proof of Theorem II (i) as was used to obtain Theorem II (ii) may be used to extend Theorem III (i) to Theorem III (ii).

LEMMA I. For $\left\{y_{t}(\omega), 0 \leqq t \leqq T^{\prime}\right\}$ a non-negative separable martingale process, $y_{t_{1}}(\omega)=0$ for almost all $\omega$ such that $\inf _{0 \leqq t \leqq t_{1}} y_{t}(\omega)=0$.

Proof. It was shown by Ville [1] (see also Doob [1, Chapter VII]) that for a discrete parameter martingale process $y_{i}(\lambda), i=1,2, \cdots$,

$$
\lambda P\left(\inf _{i=1,2, \cdots, n} y_{j}(\omega) \leqq \lambda\right) \geqq \int_{\left\{\text {inf }_{i-1,2, \cdots, n} y_{i}(\omega) \leqq \lambda\right\}} y_{n}(\omega) d P .
$$

Then for a separable martingale process $\left\{y_{t}(\omega), 0 \leqq t \leqq T^{\prime}\right\}$

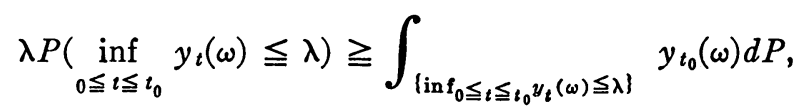

since the sets $\left\{\inf _{0 \leqq t \leqq t_{0}} y_{t}(\omega) \leqq \lambda\right\}$ can be approximated by sets defined by conditions on $y_{t}(\omega)$ for a finite number of values of $t$ in the separable case. Taking $\lambda=0$, we have

$$
0 \geqq \int_{\text {inf } \left._{0} \leqq_{t} \leqq_{t_{0}} y_{t}(\omega) \leqq 0\right\}} y_{t_{0}}(\omega) d P .
$$

Since the integral of a positive function can be zero only in case the function is almost everywhere zero, the lemma is proved.

Theorem IV. (i) $\mathrm{C}(u, u, w, o(1))$ and $\mathrm{R}(\infty)(\mathrm{R}(-\infty))$ imply that almost all sample functions are bounded from above (below).

(ii) $\mathrm{D}(u, u,, b)$ and $\mathrm{R}(\infty)(\mathrm{R}(-\infty))$ imply that almost all sample functions are bounded from above (below).

Proof. We let $I_{m}=(-m, m), m$ an integer, and consider the martingale processes $\left\{\tilde{P}\left(t, x_{t}(\omega) ; r^{\prime}, I_{m}\right), 0 \leqq t \leqq r^{\prime}\right\}$ for all $r^{\prime} \in R$. We delete the exceptional set of Lemma 1 for each of our martingale processes for each $t_{1} \in R$ from $\Omega^{*}$. The remaining set has probability one since we have deleted only a countable number of sets of measure zero. We delete also the sets where $\left|x_{r}(\omega)\right|=\infty$, for each $r$. The remaining set we call $\Omega^{* *}$, and since we have deleted only a countable number of sets of measure zero, $P\left(\Omega^{* *}\right)=1$. We assume $\mathrm{C}(u, u, w, o(1))$. We suppose an $\omega \in \Omega^{* *}$ such that for some $t_{0}$ there is a sequence $r_{i}, r_{i} \rightarrow t_{0}$ as $i \rightarrow \infty$, for which $x_{r_{i}}(\omega) \rightarrow \infty$ as $i \rightarrow \infty$. Given any $\epsilon>0$, by $\mathrm{C}(u, u, w, o(1))$ we can choose $r^{\prime}>t_{0}$, so near $t_{0}$ that, for $t \in\left(2 t_{0}-r^{\prime}, r^{\prime}\right)$, $P\left(t, x ; r^{\prime}, I(x, a(x))\right)>1-\epsilon$ for some $a(x)$ such that $|x \pm a(x)| \rightarrow \infty$ as $x \rightarrow \infty$. We assume $\mathrm{R}(\infty)$, i.e., for some $r^{\prime \prime}>r^{\prime}, P\left(r^{\prime}, x ; r^{\prime \prime}, I\right) \rightarrow 0$ as $x \rightarrow \infty$ for all bounded intervals $I$. Then for $i$ so large that $r_{i} \in\left(2 t_{0}-r^{\prime}, r^{\prime}\right)$, 


$$
\begin{aligned}
P\left(r_{i}, x_{r_{i}}(\omega) ; r^{\prime \prime}, I\right) & =\int_{-\infty}^{\infty} P\left(r_{i}, x_{r_{i}}(\omega) ; r^{\prime}, d y\right) P\left(r^{\prime}, y ; r^{\prime \prime}, I\right) \\
& \leqq \sup _{y \in I\left(x_{r_{i}}, a\left(x_{r_{i}}\right)\right)} P\left(r^{\prime}, y ; r^{\prime \prime}, I_{m}\right)+\epsilon .
\end{aligned}
$$

But by $R(\infty)$,

$$
\lim _{\mathfrak{l} \rightarrow \infty, \nu \in I\left(x_{r_{i}}(\omega), a\left(x_{r_{i}}(\omega)\right)\right)} P\left(r^{\prime}, y, r^{\prime \prime}, I_{m}\right) \leqq \limsup _{y \rightarrow \infty} P\left(r^{\prime}, y, r^{\prime \prime}, I_{m}\right)=0 .
$$

Hence, since $\epsilon$ was arbitrary,

$$
\lim _{i \rightarrow \infty} P\left(r_{i}, x_{r_{i}}(\omega) ; r^{\prime \prime}, I_{m}\right)=0 .
$$

Applying Lemma I, we have $P\left(r^{\prime \prime}, x_{r^{\prime \prime}}(\omega) ; r^{\prime \prime}, I_{m}\right)=0$. Since we have eliminated the exceptional sets of Lemma $I$, we must have $\left|x_{r^{\prime \prime}}(\omega)\right|>m$. This holds however for all $m$, so $\left|x_{r^{\prime \prime}}(\omega)\right|=\infty$. This contradicts the construction of $\Omega^{* *}$. A similar argument holds using $\mathrm{R}(-\infty)$. Hence (i) follows.

The same proof establishes (ii).

TheOREM V. C $(u, u, a, o(1))$ implies that the sample functions are bounded and have no discontinuities with oscillation exceeding $4 a$ other than jumps, with probability one.

Proof. Since we have assumed the $x_{t}(\omega)$ process to have property $\mathrm{S}^{*}$, it will be sufficient to establish that the theorem holds when we assume the approach to $t$ from above and below through points of $R$.

Since $P(t, x ; s, I(x, a)) \rightarrow 1$ as $t-s \downarrow 0$, uniformly in $t$, we can pick $\delta$ so small that $s-t<\delta$ implies that $P(r, x ; s, I(x, a))>1-\epsilon, r \in(t, s)$. We pick $(s, t)$ so that $s-t<\delta, s, t$, taken from $R$. This interval we divide into a net,

$$
T_{n}=\left(t=t_{0} ; t_{1}, t_{2}, \cdots, t_{n}=s\right), \quad t_{i} \in R .
$$

We consider the following sets:

$$
\begin{aligned}
\mu_{a} & =\left\{\omega|| x_{t}(\omega)-x_{s}(\omega) \mid<a\right\}, \\
\Lambda_{j} & =\bigcap_{i=1}^{i=j-1}\left\{\omega|| x_{t_{i}}(\omega)-x_{t}(\omega) \mid \leqq 2 a\right\} \cap\left\{\omega|| x_{t_{j}}(\omega)-x_{t}(\omega) \mid>2 a\right\}, j<n, \\
\Lambda_{j}^{\prime} & =\left\{\omega|| x_{t_{j}}(\omega)-x_{s}(\omega) \mid>a\right\} .
\end{aligned}
$$

We note that for $i \neq j, \Lambda_{i} \cap \Lambda_{j}=0$. Since we must have $\left|x_{t_{i}}(\omega)-x_{8}(\omega)\right|$ $>a$ if $\omega \in \mu_{a} \cap \Lambda_{j}$,

$$
\mu_{a} \subset \bigcup_{j=1}^{j=n-1}\left(\Lambda_{j} \cap \Lambda_{j}^{\prime}\right) \cup \mathrm{c} \bigcup_{j=1}^{j=n-1} \Lambda_{j}
$$

(We use $c \Lambda$ to denote the complement of $\Lambda$.) Since the $\Lambda_{j}$ are disjunct, we have 


$$
\begin{aligned}
P\left(\mu_{a}\right) & \leqq \sum_{j=1}^{j=n-1} P\left(\Lambda_{j} \cap \Lambda_{j}^{\prime}\right)+P\left(\mathrm{c} \bigcup_{j=1}^{j=n-1} \Lambda_{j}\right) \\
& =\sum_{j=1}^{j=n-1} P\left(\Lambda_{j} \cap \Lambda_{j}^{\prime}\right)+1-P\left(\bigcup_{j=1}^{j=n-1} \Lambda_{j}\right) .
\end{aligned}
$$

Since the $\Lambda_{j}$ are defined by conditions on $x_{t_{0}}(\omega), x_{t_{1}}(\omega), \cdots, x_{t_{j}}(\omega)$, we may use the Markov property and $\mathrm{C}(u, u, a, o(1))$ to obtain

$$
\begin{aligned}
P\left(\Lambda_{j} \cap \Lambda_{j}^{\prime}\right) & =\int_{\Lambda_{j}} P\left(\Lambda_{j}^{\prime} \mid x_{t_{1}}(\omega)=x_{j}, \cdots, x_{t_{0}}(\omega)=x_{0}\right) d P \\
& =\int_{\Lambda_{j}} P\left(\Lambda_{j}^{\prime} \mid x_{t_{j}}(\omega)=x_{j}\right) d P \\
& \leqq \sup _{x} P\left(\Lambda_{j}^{\prime} \mid x_{t_{j}}(\omega)=x\right) P\left(\Lambda^{\prime}\right) \\
& \leqq \epsilon P\left(\Lambda_{j}\right) .
\end{aligned}
$$

Hence

$$
\begin{aligned}
P\left(\mu_{a}\right) & \leqq \epsilon \sum_{j=1}^{j=n-1} P\left(\Lambda_{j}\right)+1-P\left(\bigcup_{j=1}^{j=n-1} \Lambda_{j}\right) \\
& =\epsilon P\left(\bigcup_{j=1}^{j=n-1} \Lambda_{j}\right)+1-P\left(\bigcup_{j=1}^{j=n-1} \Lambda_{j}\right) .
\end{aligned}
$$

Since $P\left(\mu_{a}\right)>1-\epsilon$,

$$
P\left(\bigcup_{j=1}^{j=n-1} \Lambda_{j}\right) \leqq \epsilon /(1-\epsilon)
$$

We note that this inequality is independent of $n$, or of the particular choice of $T_{n}$. We let $T_{n}$ approach $R$ by adding to the net more and more points from $R$.

${ }_{n} \Lambda^{1}=\bigcup_{j=1}^{j=n-1} \Lambda_{j}$ is a measurable set. Since $T_{n+1}$ includes all the points of $T_{n, n+1} \Lambda^{1} \supset_{n} \Lambda^{1}$. Hence $\lim { }_{n} \Lambda^{1}$ exists and is a measurable set. We call it $\Lambda^{1} \cdot{ }_{n} \Lambda^{1}$ for every $n$ includes all $\omega$ for which $\left|x_{t_{i}}(\omega)-x_{t_{j}}(\omega)\right|>4 a, i, j<n-1, t_{i}, t_{j} \in T_{n}$. Going to the limit in the above inequality, we have

$$
P\left(\Lambda^{1}\right) \leqq \epsilon /(1-\epsilon) .
$$

When we say that $x_{t}(\omega)$ has experienced $k$ displacements of more than $4 a$ on $T_{n}$, we mean that there exist $k$ pairs of values $\left(t_{i_{j}}, t_{i_{j}+\mu_{j}}\right)$ taken from $T_{n}$, with $t_{i_{j+1}}>t_{i_{j}+\mu_{j}}$, such that $\left|x_{i_{i j}}(\omega)-x_{i_{i j}+\mu_{j}}(\omega)\right|>4 a, j=1,2, \cdots, k$. We define: $\mu_{k a}=\left\{\omega \mid x_{t}(\omega)\right.$ has experienced $k$ or more displacements of more than $4 a$ on the net $T_{n}$ up to $\left.t_{n-1}\right\}$.

$\Lambda_{k i a}=\left\{\omega \mid x_{t}(\omega)\right.$ has experienced $k$ displacements of more than $4 a$ on the net $T_{n}$ up to $t_{i-1}$, but not before $\}$. 
$\Lambda_{i}^{\prime} a=\left\{\omega \mid x_{t}(\omega)\right.$ experiences at least one displacement of more than $4 a$ on $T_{n}$ between $t_{i-1}$ and $\left.t_{n-1}\right\}$.

We note that $\Lambda_{k_{i}}{ }_{a} \cap \Lambda_{k_{j} a}=0, i \neq j, \sum_{i=1}^{i=n} \Lambda_{k_{i} a}=\mu_{k a}$ and

$$
\mu_{k+1} a=\bigcup_{i=1}^{i=n}\left(\Lambda_{k i} a \cap \mu_{i a}^{\prime}\right) \text {. }
$$

Since the $\Lambda_{k i \text { a }}$ are disjunct,

$$
P\left(\mu_{k+1} a\right)=P\left(\bigcup_{i=1}^{i=n} \Lambda_{k i a} \cap \mu_{i a}^{\prime}\right)=\sum_{i=1}^{i=n} P\left(\Lambda_{k i a} \cap \Lambda_{i a}^{\prime}\right) .
$$

Since $\Lambda_{k i \text { a }}$ is defined by conditions on $x_{t_{0}}(\omega), x_{t_{1}}(\omega), \cdots, x_{t_{i-1}}(\omega)$, we may use the Markov property to obtain

$$
\begin{aligned}
P\left(\Lambda_{k i a} \cap \Lambda_{i a}^{\prime}\right) & =\int_{\Delta k i a} P\left(\Lambda_{i a}^{\prime} \mid x_{t_{i-1}}(\omega)=x_{i-1}, \cdots, x_{t_{0}}(\omega)=x_{0}\right) d P \\
& =\int_{\Delta k i a} P\left(\Lambda_{i a}^{\prime} \mid x_{t_{i-1}}(\omega)=x_{i-1}\right) d P \\
& \leqq \sup _{x} P\left(\Lambda_{i a}^{\prime} \mid x_{t_{i-1}}(\omega)=x\right) P\left(\Lambda_{k i a}\right) .
\end{aligned}
$$

Hence, applying the first part of the proof to $\Lambda_{i}^{\prime}$ a, we have

$$
P\left(\Lambda_{k i a} \cap \Lambda_{i a}^{\prime}\right) \leqq \epsilon P\left(\Lambda_{k i a}\right) /(1-\epsilon),
$$

so

$$
P\left(\mu_{k+1} a\right) \leqq \epsilon \sum_{i=1}^{i=n} P\left(\Lambda_{k i} a\right) /(1-\epsilon)=\epsilon P\left(\mu_{k a}\right) /(1-\epsilon) .
$$

Therefore, by induction, for all $k$,

$$
P\left(\mu_{k} a\right) \leqq(\epsilon /(1-\epsilon))^{k} .
$$

We let $\Lambda^{k}=\lim _{n \rightarrow \infty} \mu_{k a}$. Again taking limits on the inequality, we have

$$
P\left(\Lambda^{k}\right) \leqq(\epsilon /(1-\epsilon))^{k} .
$$

Since

$$
\sum_{k=1}^{k=\infty} P\left(\Lambda^{k}\right) \leqq \sum_{k=1}^{k=\infty}(\epsilon /(1-\epsilon))^{k}=\epsilon /(1-2 \epsilon),
$$

we may apply the Borel-Cantelli lemma to establish that, with probability one, $\omega$ is in but a finite number of the $\Lambda^{k}$. That is, on the interval $(t, s)$, the number of displacements of more than $4 a$ is finite, with probability one. We cover $\left(0, T^{\prime}\right)$ by a finite number of intervals of length $\delta$ or less, end points taken from $R$. If we eliminate the exceptional set for each, we eliminate only 
a set of measure zero. The remaining set we call $\mu . P(\mu)=1$. For $\omega \in \mu$, if the number of displacements exceeding $4 a$ is infinite on $\left(0, T^{\prime}\right)$, it must be so on at least one member of the covering. Hence the number of displacements of more than $4 a$ on $\left(0, T^{\prime}\right)$ is finite, with probability one. This establishes the theorem.

We have as a corollary:

Corollary. $\mathrm{C}(u, u, \epsilon, o(1))$ implies that almost all sample functions are continuous except for strict jumps.

TheOREM VI. (i) $\mathrm{D}(u, b, \quad, b)$ implies that for almost all $\omega$, for all $t$, $0 \leqq t \leqq T^{\prime}$,

$$
\lim _{s \uparrow l,(s \downarrow t)} \frac{x_{s}(\omega)-c}{1+x_{s}^{2}(\omega)}=0 .
$$

(c depends on $\omega, t$, and the direction of approach.)

(ii) $\mathrm{D}(u, u,, b)$ implies that for almost all $\omega$, if $\lim _{\inf _{s} \uparrow t(s \downarrow t)}\left|x_{s}(\omega)\right|<\infty$, then $\lim \sup _{s \uparrow t(s \downarrow t)}\left|x_{s}(\omega)\right|<\infty$.

From (i) and (ii) $\mathrm{D}(u, u,, b)$ implies that for almost all $\omega$, $\arctan x_{t}(\omega)$, $\bmod \pi$, is continuous except for strict jumps.

(iii) $\mathrm{D}(u, u,, u)$ implies that almost all sample functions have right- and left-hand limits (possibly infinite) at every $t$. Since we deal with processes with property $\mathrm{S}^{*}$, this implies that $\arctan x_{t}(\omega)$ is continuous except for strict jumps.

(iv) $\mathrm{D}(u, b,, b), \mathrm{R}(\infty), \mathrm{R}(-\infty)$ and $\mathrm{C}(u, u, w, o(1))$ imply that almost all sample functions are continuous except for strict jumps.

(v) $\mathrm{C}(u, b, \epsilon, o(1))($ or $\mathrm{D}(u, b,, b))$ and $\mathrm{C}(u, u, a, o(1))$ imply that almost all sample functions are continuous except for strict jumps.

Proof of (i). We suppose that there is an $\omega \in \Omega^{*}$ for which there exists a $t_{0}$ such that there is a sequence $r_{i}, r_{i} \uparrow t_{0}$, and a sequence $r_{i}^{\prime}, r_{i}^{\prime} \uparrow t_{0}\left(r_{i} \downarrow t_{0}\right.$, $\left.r_{i}^{\prime} \downarrow t_{0}\right)$, for which $x_{r_{i}}(\omega) \rightarrow a, x_{r_{i}}(\omega) \rightarrow b, a \neq b, a, b$ finite. We choose $I, A$ as in the proof of Theorem II (i). Under the assumption $\mathrm{D}(u, b,, b)$ we can choose $r^{\prime}, r^{\prime}>t_{0}$, so near $t_{0}$ that for $t \in\left(2 t_{0}-r^{\prime}, r^{\prime}\right), P\left(t, x ; r^{\prime}, I\right)>0.9, x \in A \cap I$, $P\left(t, x ; r^{\prime}, I\right)<0.1, x \in A \cap c I$. Then for $j$ so large that for $j>1, r_{j} \in\left(2 t_{0}-r^{\prime}, r^{\prime}\right)$, $r_{j}^{\prime} \in\left(2 t_{0}-r^{\prime}, r^{\prime}\right), x_{r_{j}}(\omega) \in A \cap I, x_{r_{j}^{\prime}}^{\prime}(\omega) \in A \cap c I$, we have, for $j>i$,

$$
P\left(r_{j}, x_{r_{j}}(\omega) ; r^{\prime}, I\right)>0.9, \quad P\left(r_{j}^{\prime}, x_{r_{j}}(\omega) ; r^{\prime}, I\right)<0.1 .
$$

This implies that $\tilde{P}\left(t, x_{t}(\omega) ; r^{\prime}, I\right)$ has an oscillatory discontinuity at $t_{0}$, contrary to the construction of $\Omega^{*}$. Hence we have proved that for $\omega \in \Omega^{*}$, $x_{r}(\omega)$ can have at most one finite limiting value from the right (left) at each value of $t$. Since the process has property $\mathrm{S}^{*}$, the same must be true of $x_{t}(\omega)$. This limiting value is the " $c$ " of (i).

Proof of (ii). We suppose an $\omega \in \Omega^{*}$ for which a $t_{0}$ exists such that there is a sequence $r_{i}, r_{i} \uparrow t_{0}$ as $i \rightarrow \infty$, and a sequence $r_{i}^{\prime}, r_{i}^{\prime} \uparrow t_{0}$ as $i \rightarrow \infty\left(r_{i} \downarrow t_{0}\right.$, 
$\left.r_{i}^{\prime} \downarrow t_{0}\right)$, for which $x_{r_{i}}(\omega) \rightarrow a, a \neq \pm \infty,\left|x_{r_{i}}(\omega)\right| \rightarrow \infty$. We choose $I, A$ as in the proof of Theorem II (ii), $r^{\prime}$ as above. An argument identical with that above, with $\mathrm{D}(u, u, \quad, b)$ insuring that $\left.\left.P\left(r_{j}^{\prime}, x_{r_{i}}^{\prime}\right) \omega\right) ; r^{\prime}, I\right)<0.1$ for $j$ sufficiently large, shows $\tilde{P}\left(t, x_{t}(\omega) ; r^{\prime}, I\right)$ to have an oscillatory discontinuity, contrary to the construction of $\Omega^{*}$. An argument like that above shows that this implies the result for limits taken through $R$, and since the process has property $\mathrm{S}^{*}$, the result follows for limits taken through all values of $t$.

Proof of (iii). From (i) and (ii) we see that under the hypothesis $\mathrm{D}(u, u, \quad, u)$, the only possibility that $\lim _{r \uparrow t(r \downarrow t)} x_{r}(\omega)$ not exist for all $\omega \in \Omega^{*}$, all $t$, is that, for some $t_{0}, \lim \inf _{r \uparrow t_{0}\left(r \downarrow t_{0}\right)} x_{r}(\omega)=-\infty, \lim \sup _{r \uparrow t_{0}\left(r \downarrow t_{0}\right)} x_{r}(\omega)=\infty$. We choose $r_{i}$ and $r_{i}^{\prime}$ to be sequences along which these limiting values are taken. $I$ we choose to be $(0, \infty), A$ to be the real line, less the interval $(-1,1)$. Then $\mathrm{D}(u, u, \quad, u)$ assures that we can choose $r^{\prime}$ as before. With this choice of sequences and martingale process, the argument of (i) holds exactly as before.

(iv) and (v) follow immediately from (i) and Theorem IV.

The results of Theorem VI (ii) were obtained by Doob [4, Theorem 12] for the case where $x_{t}(\omega)$ can take on integral values. His hypotheses are very close to $\mathrm{D}(u, u, \quad, b)$.

Doeblin, making no restriction on the number of elements in his space or on its topological structure, under the assumption that

$$
P(t, x ; t+h,\{x\}) \rightarrow 1 \text { as } h \rightarrow 0
$$

uniformly in $t$ and $x$, proved that almost all sample functions of his process were continuous in finite closed intervals except at a finite number of values of $t$ and constant between successive pairs of these values. His assumption is very similar to $\mathrm{C}(u, u, \epsilon, o(1))$. In the corollary to Theorem $\mathrm{V}$ we have the analogous result. Processes whose sample functions are continuous except for jumps, but have an infinite number of jumps, in finite closed intervals, do exist, as Lévy [2] has shown in his discussion of the infinitely divisible processes. Hence we cannot expect as strong a result as that of Doeblin in this case.

3. Continuity of sample functions. Thus far the hypotheses used do not prevent jumps in the sample functions. This should be the case, since the Poisson process satisfies all the hypotheses used so far. It does not, however, satisfy $\mathrm{C}(u,, \epsilon, o(h))$. We first use $\mathrm{C}(u, u, \epsilon, o(h))$. Without loss of generality we may take $T^{\prime}=1$. We examine the behavior of $x_{t}(\omega)$ on a net, $T_{n}$ $=\left(0, t_{1}^{n}, \cdots, t_{2^{n-1}}^{n}, 1\right)$, where $t_{i}^{n}=i / 2^{n}$. We let $d_{n i}=\left[t_{i-1}^{n}, t_{i}^{n}\right], \Delta_{n i}=\mid x_{t_{i}^{n}}^{n}(\omega)$ $-x_{t i-1}^{n}(\omega) \mid$.

We observe

$$
P\left(\max _{i=1,2, \cdots, 2^{n}} \Delta_{n i}>\epsilon\right) \leqq \sum_{i=1}^{i=2^{n}} P\left(\Delta_{n i}>\epsilon\right) .
$$

Using the Markov property, we have 


$$
\begin{aligned}
P\left(\Delta_{n i}>\epsilon\right) & =E\left\{P\left(\Delta_{n i}>\epsilon \mid x_{(i-1) / 2^{n}}(\omega)=x\right)\right\} \\
& \leqq \sup _{x} P\left(\Delta_{n i}>\epsilon \mid x_{(i-1) / 2^{n}}(\omega)=x\right) .
\end{aligned}
$$

Hence, using $\mathrm{C}(u, u, \epsilon, o(h))$, we have

$$
P\left(\max _{i=1,2, \cdots, 2^{n}} \Delta_{n i}>\epsilon\right)=2^{n} o\left(1 / 2^{n}\right)=o(1)_{(n \rightarrow \infty)} .
$$

For every $t$, there is a sequence of meshes, $d_{n i_{t}}$, of the successive nets such that $t \in d_{n i_{t}}$ and $d_{n i_{t}} \subset d_{n-1}{ }_{i t}$, for all $n$. We select a sequence $n_{j}$ such that the left-hand side of (3) is for each $i$ a member of a convergent series. Applying the Borel-Cantelli lemma to this series, we may deduce that, with probability one, for $j$ sufficiently large,

$$
\max _{i=1,2, \cdots, 2^{n_{j}}} \Delta_{n i}<\epsilon .
$$

The left-hand end point of $d_{n_{j} i_{i}}$ we call $s_{j}$, the right-hand end point $t_{j}$. It follows from (4) that:

LEMMA II. C $(u, u, \epsilon, o(h))$ implies that for almost all sample functions there exist sequences $t_{i} \downarrow t, s_{i} \uparrow t$, for each $t$, such that

$$
\lim _{i \rightarrow \infty}\left|x_{t_{i}}(\omega)-x_{s_{i}}(\omega)\right|=0 \text {. }
$$

Similar properties of bounded sample functions can be obtained under weaker hypotheses, namely $\mathrm{C}(u, b, \epsilon, o(h))$, by a similar calculation. Using the same net, we have

$$
\begin{aligned}
P\left(\max _{i=1,2, \cdots, 2^{n}} \Delta_{n i}>\epsilon, \sup _{0 \leqq s \leqq 1} \mid\right. & \left.x_{8}(\omega) \mid \leqq M\right) \\
& \leqq \sum_{i=1}^{i=2^{n}} P\left(\Delta_{n i}>\epsilon, \sup _{0 \leqq s \leqq(i-1) / 2^{n}}\left|x_{8}(\omega)\right| \leqq M\right) .
\end{aligned}
$$

Using the Markov property,

$$
\begin{aligned}
& P\left(\Delta_{n i}>\epsilon, \sup _{0 \leqq s \leqq(i-1) / 2^{n}}\left|x_{8}(\omega)\right| \leqq M\right)
\end{aligned}
$$

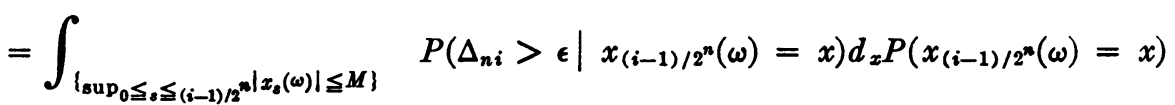

$$
\begin{aligned}
& \leqq \sup _{|x| \leqq M} P\left(\Delta_{n i}>\epsilon \mid x_{(i-1) / 2^{n}}(\omega)=x\right) \text {. }
\end{aligned}
$$

Using $\mathrm{C}(u, b, \epsilon, o(h))$, we have

$$
P\left(\Delta_{n i}>\epsilon, \sup _{0 \leqq s \leqq(i-1) / 2^{n}}\left|x_{s}(\omega)\right|<M\right)=o\left(1 / 2^{n}\right) .
$$

Hence 


$$
P\left(\max _{i=1,2, \ldots, 2^{n}} \Delta_{n i}>\epsilon, \sup _{0 \leqq s \leqq 1}\left|x_{s}(\omega)\right| \leqq M\right)=2^{n} o\left(1 / 2^{n}\right)=o(1)_{(n \rightarrow \infty)} .
$$

By an argument similar to that of Lemma II, since this equation holds for all $M$, we can state:

LEMMA III. C $(u, b, \epsilon, o(h))$ implies that for almost all bounded sample functions, $x_{t}(\omega)$, there exist sequences, $t_{i} \downarrow t, s_{i} \uparrow t$, for each $t$, such that

$$
\lim _{i \rightarrow \infty}\left|x_{t_{i}}(\omega)-x_{s_{i}}(\omega)\right|=0 \text {. }
$$

Since $\mathrm{C}(u, u, \epsilon, o(h))$ implies $\mathrm{D}(u, u, \quad, u)$, Theorem VI, the corollary to Theorem V, Lemma II, and Lemma III yield the theorem:

Theorem VII. (i) $\mathrm{C}(u, b, \epsilon, o(h))$ and $\mathrm{D}(u, u, \quad, b)$ imply that almost all bounded sample functions are continuous.

(ii) $\mathrm{C}(u, u, \epsilon, o(h))$ implies that almost all sample functions are continuous.

(iii) $\mathrm{C}(u, b, \epsilon, o(h))$ and $\mathrm{C}(u, u, a, o(1))$ imply that almost all sample functions are continuous.

(iv) $\mathrm{C}(u, b, \epsilon, o(h)), \mathrm{C}(u, u, w, o(1)), \mathrm{R}(\infty)$, and $\mathrm{R}(-\infty)$ imply that almost all sample functions are continuous.

(ii) is similar to a result of Doeblin [3], announcing conditions under which $x_{t}(\omega) /\left(1+\left|x_{t}(\omega)\right|\right)$ is continuous. He assumes, essentially, $\mathrm{C}(u, b, \epsilon, o(h))$, conditions resembling $\mathrm{R}(\infty)$ and $\mathrm{R}(-\infty)$, and the existence of continuous first and second moments of the increments of $x_{t}(\omega)$. Since he announces other results from his hypotheses, it is not certain which of them were used to obtain this result.

Lemma III can be used in another way. Doob [1, Chapter VI, Theorem 3.2] has the theorem:

ThEOREM. If $\left\{x_{t}(\omega), a \leqq t \leqq b\right\}$ is a separable stochastic process with the following properties:

(a) The process is measurable,

(b) $E\left\{\left|x_{t}(\omega)\right|\right\}<\infty, a \leqq t \leqq b: \int_{a}^{b} E\left\{\left|x_{t}(\omega)\right|\right\} d t<\infty$,

(c) there is a Baire function, $m(t, \xi)$, with $m(t, \xi)<K\left(1+\xi^{2}\right)^{1 / 2}$ for some constant $K$, such that, if $a \leqq t_{1}<t_{2} \leqq b$,

$$
E\left\{x_{t_{1}}(\omega)-x_{t_{2}}(\omega) \mid x_{t}(\omega), t \leqq t_{1}\right\}=E\left\{\int_{t_{1}}^{t_{2}} m\left(s, x_{s}(\omega)\right) d s \mid x_{t}(\omega), t<t_{1}\right\}
$$

with probability one. Then $X_{t}(\omega)$, defined by

$$
X_{t}(\omega)=x_{t}(\omega)-\int_{a}^{t} m\left(s, x_{s}(\omega)\right) d s, \quad a \leqq t \leqq b,
$$

is a separable martingale process and in fact, if $t_{1}<t_{2}$, 


$$
E\left\{X_{t_{2}}(\omega) \mid x_{t}(\omega), t<t_{1}\right\}=X_{t_{1}}(\omega)
$$

with probability one.

If we use the theorem of Doob cited in the introduction, since $X_{t}(\omega)$ is a separable martingale process, it has sample functions which are bounded and have finite right- and left-hand limits for all $t$, with probability one. Since $\int_{a}^{t} m\left(s, x_{s}(\omega)\right) d s$ is a bounded continuous function, the function $x_{t}(\omega)$ must have the same continuity properties as the function $X_{t}(\omega)$, with probability one. Applying this result to our case, using also Lemma III and the property $\mathrm{S}^{*}$, we have the theorem:

THEOREM VIII. The sample functions of a Markov process with property $\mathrm{S}^{*}$ whose transition probability functions satisfy the conditions (a), (b), and (c) of the introduction, condition $\mathrm{C}(u, b, \epsilon, o(h))$ and the conditions of the above theorem, are almost all continuous.

The conditions of the quoted theorem are similar to the conditions imposed on $P(t, x ; T, X)$ and the first moment of the increment used to insure that $P(t, x ; T, X)$ satisfies the Fokker-Planck equations.

4. Examples. We first show that Theorem VI furnishes a proof that almost all sample functions of the Brownian motion process are continuous. The function satisfying (a), (b), and (c) of the introduction is

$$
P(t, x ; T, X)=\int_{X} \frac{e^{-(y-x)^{2} / 2(T-t)}}{(2 \pi(T-t))^{1 / 2}} d y \text {. }
$$

Then we have

$$
\begin{aligned}
1-P(t, x ; t+h, I(x, \epsilon)) & =\int_{|y-x|>c} \frac{e^{-(y-x)^{2} / 2 h}}{(2 \pi h)^{1 / 2}} d y=2 \int_{\epsilon}^{\infty} \frac{e^{-u^{2} / 2 h}}{(2 \pi h)^{1 / 2}} d u \\
& \leqq \frac{1}{\epsilon} \int_{\epsilon}^{\infty} \frac{2 u e^{-u^{2} / 2 h}}{(2 \pi h)^{1 / 2}} d u=\frac{e^{-\epsilon^{2} / 2 h}}{\epsilon \pi^{1 / 2}}=o(h)_{(h \rightarrow 0)}
\end{aligned}
$$

uniformly in $x$, for all $\epsilon$. Hence $\mathrm{C}(u, u, \epsilon, o(h))$ is satisfied. Then, by Theorem VII, (ii), almost all the sample functions of the Brownian motion process are continuous.

We now construct a number of examples which show that discontinuities can occur when various of our hypotheses are not satisfied. We suppose given a Brownian motion process $\left\{x_{t}(\omega), 0 \leqq t \leqq T^{\prime}\right\}, x_{0}(\omega)=0, \alpha=1$. For each $t$, we define $\tilde{x}_{t}(\omega)$ as follows:

$$
\tilde{x}_{t}(\omega)=1-\left|x_{t}(\omega)-1\right| \text {. }
$$

We let $V=(x \mid x<\gamma) \cup(x \mid x>2-\gamma)$. For each $\gamma \leqq 1,\left(\omega \mid \bar{x}_{t}(\omega)<\gamma\right)=\left(\omega \mid x_{t}(\omega)\right.$ $\in V)=\left(\omega \mid x_{t}(\omega)<\gamma\right) \cup\left(\omega \mid x_{t}(\omega)>2-\gamma\right)$; for $\gamma \geqq 1,\left(\omega \mid \tilde{x}_{t}(\omega) \leqq \gamma\right)=\Omega$. There- 
fore $\tilde{x}_{t}(\omega)$ is a measurable function, for each $t$, so $\left\{\tilde{x}_{t}(\omega), 0 \leqq t \leqq T^{\prime}\right\}$ is a stochastic process. The transformation (5) preserves the continuity of sample functions. The functions satisfying the conditions (a), (b), and (c) for the $x_{t}(\omega)$ process given in the introduction are, for $V$ described above,

$$
P(t, x ; T, V)=\int_{-\infty}^{\gamma} \frac{e^{-(y-x)^{2} / 2(T-t)}}{(2 \pi(T-t))^{1 / 2}} d y+\int_{2-\gamma}^{\infty} \frac{e^{-(y-x)^{2} / 2(T-t)}}{(2 \pi(T-t))^{1 / 2}} d y .
$$

By direct calculation we have

$$
P(t, x ; T, V)=P(t, 2-x ; T, V) .
$$

Hence, letting $\tilde{P}(t, x ; T, X)$ be the transition probability function of the $\tilde{x}_{t}(\omega)$ process, $I=(x \mid x<\gamma)$, we have $\widetilde{P}(t, x ; T, I)=P(t, x ; T, V)$. Then, letting $t=t_{0}>t_{1}>\cdots>t_{n}$, and using the Markov property of the $x_{t}(\omega)$ process, we have

$$
\begin{aligned}
P & \left\{\tilde{x}_{T}(\omega)<\gamma \mid \bar{x}_{t_{i}}(\omega), i=0,1, \cdots, n\right\} \\
& =P\left\{x_{T}(\omega) \in V \| x_{t_{i}}(\omega)-1 \mid, i=0,1, \cdots, n\right\} \\
& =E\left[P\left\{x_{T}(\omega) \in V \mid x_{t_{i}}(\omega), i=0,1, \cdots, n\right\}|| x_{t_{i}}(\omega)-1 \mid, i=0,1, \cdots, n\right] \\
& =E\left[P\left\{x_{T}(\omega) \in V \mid x_{t_{0}}(\omega)\right\}|| x_{t_{i}}(\omega)-1 \mid, i=0,1, \cdots, n\right] .
\end{aligned}
$$

But from the equation nine lines above, the last line is simply

$$
P\left\{x_{T}(\omega) \in V \mid \tilde{x}_{t_{0}}(\omega)\right\} \text {. }
$$

So

$$
\begin{aligned}
P\left\{x_{T}(\omega) \in V \mid x_{t_{0}}(\omega)\right\} & =P\left(x_{T}(\omega) \in V \mid x_{t_{0}}(\omega)\right) \\
& =P\left\{\bar{x}_{T}(\omega)<\gamma \mid x_{t_{0}}(\omega)\right\} .
\end{aligned}
$$

Hence the $\tilde{x}_{t}(\omega)$ process is also a Markov process.

From the $\tilde{x}_{t}(\omega)$ process we form the $y_{t}(\omega)$ process as follows: for each $\omega$ we let

$$
\begin{array}{lr}
y_{t}(\omega)=\tilde{x}_{t}(\omega) & \text { for } x_{t}(\omega)<0, \\
y_{t}(\omega)=\tan \left(\pi x_{t}(\omega) / 2\right) & \text { for } 0 \leqq x_{t}(\omega) \leqq 1 .
\end{array}
$$

The $y_{t}(\omega)$ process is also a Markov process. For almost all $\omega$, at any value of $t$ for which $\tilde{x}_{t}(\omega) \neq 1, y_{t}(\omega)$ is continuous, since the transformation (6) is continuous for $x_{t}(\omega) \neq 1$. However for $\omega, t_{0}$ such that $\lim _{t \rightarrow t_{0}} x_{t}(\omega)=1$, $\lim _{t \rightarrow t_{0}} y_{t}(\omega)=\infty$. That the transition probability functions of $\left\{y_{t}(\omega)\right.$, $\left.0 \leqq t \leqq T^{\prime}\right\}$ satisfy $\mathrm{C}(u, b, \epsilon, o(h))$ can be seen by a calculation very similar to that used in showing that the transition probability functions of the Brownian motion process satisfy $\mathrm{C}(u, u, \epsilon, o(h))$. However, neither $\mathrm{R}(\infty)$ nor $\mathrm{C}(u, u, a, o(1))$ is satisfied. To show this, we take $I$ to be the interval $(-1,0)$. For $y_{t}(\omega) \rightarrow \infty$, we have $x_{t}(\omega) \rightarrow 1$ in the original process. If $\bar{P}(t, y ; T, I)$ 
is the transition probability function of the $y_{t}(\omega)$ process, we have

$$
\lim _{y \rightarrow \infty} \bar{P}(t, y ; T, I)=\int_{-1}^{0} \frac{e^{-(x-1)^{2} / 2(T-t)}}{(2 \pi(T-t))^{1 / 2}} d x+\int_{2}^{3} \frac{e^{-(x-1)^{2} / 2(T-t)}}{(2 \pi(T-t))^{1 / 2}} d x .
$$

This expression is not zero, so $R(\infty)$ is not satisfied. Also

$$
\bar{P}(t, y ; T, I(y, a))=\int_{\arctan (y-a)}^{\arctan (y+a)} \frac{e^{-(x-\arctan y)^{2} / 2(T-t)}}{(2 \pi(T-t))^{1 / 2}} d x .
$$

But since $\lim _{z \rightarrow \infty} \arctan z=\pi / 2$ in the range with which we are concerned, for all $T-t>0, \lim _{y \uparrow_{\infty}} \bar{P}(t, y ; T, I(y, a))=0$. Hence it is impossible that $\lim _{T-t \downarrow o} P(t, y ; T, I(y, a))=1$ uniformly for all $y$, and so $\mathrm{C}(u, u, a, o(1))$ is not satisfied.

We now construct an example to illustrate what may happen in case $\mathrm{D}(u, u,, u), \mathrm{R}(\infty)$, and $\mathrm{R}(-\infty)$ are not satisfied, but $\mathrm{D}(u, u,, b)$ is satisfied. We suppose $\left\{x_{t}(\omega), 0 \leqq t \leqq T^{\prime}\right\}$ to be a Brownian motion process with $x_{0}(\omega)=1 / 2, \alpha=1$. We define an $\tilde{x}_{t}(\omega)$ process as follows: for each $\omega$,

$$
\tilde{x}_{t}(\omega)=x_{t}(\omega)-i, \quad \text { for } i \leqq x_{t}(\omega)<i+1 .
$$

We let $V=U_{i=-\infty}^{\{=\infty}(x \mid i \leqq x<i+\gamma), \gamma \leqq 1$. Then

$$
\left\{\omega \mid \tilde{x}_{i}(\omega)<\gamma\right\}=\bigcup_{i=-\infty}^{i=\infty}\left\{\omega \mid i \leqq x_{t}(\omega)<i+\gamma\right\} .
$$

Since this is a denumerable sum of measurable sets, $\tilde{x}_{t}(\omega)$ is for each $t$ a measurable function, and so $\left\{\tilde{x}_{t}(\omega), 0 \leqq t \leqq T^{\prime}\right\}$ is a stochastic process. From the mapping (7) we see that the sample functions of the $\tilde{x}_{t}(\omega)$ process will be almost all continuous except at values of $t$ for which $x_{t}(\omega)=i, i=\cdots$, $-1,0,1, \ldots$. By direct calculation we find

$$
P(t, x+j ; T, V)=P(t, x ; T, V) .
$$

Using the same conventions and proof as in the previous example, we may show that the $\tilde{x}_{t}(\omega)$ process is a Markov process. We consider $y_{t}(\omega)$ defined for each $\omega$ as follows:

$$
y_{t}(\omega)=\tan \left[\pi\left(x_{t}(\omega)-1 / 2\right)\right] .
$$

As before the $y_{t}(\omega)$ process is a Markov process. Since the $\tilde{x}_{t}(\omega)$ process sample functions are almost all continuous except at values of $t$ where $\tilde{x}_{t}(\omega)=0$, the only discontinuities of the sample functions of the $y_{t}(\omega)$ process, for corresponding $\omega$, are at $\infty$ and $-\infty$. However, in a $t$-neighborhood of a value $t_{0}$ of $t$ for which $x_{t_{0}}(\omega)=i, x_{t}(\omega)$ crosses the value $i$ an infinite number of times with probability one [P. Levy, 1, p. 85]. Therefore $y_{t}(\omega)$ has both $-\infty$ and $\infty$ as limits at $t_{0}$ with probability one. This indicates that $y_{t}(\omega)$ does not satisfy $\mathrm{D}(u, u,, u)$. We take $I=(0, \infty)$ and let $h_{i}=T_{i}-t, i=1,2, \cdots$. 
We let $h_{i} \rightarrow 0$. For $\mathrm{D}(u, u, \quad, u)$ to be satisfied it is necessary that $\bar{P}\left(t, y_{i} ; T_{i}, I\right)$ $\rightarrow 1$ for any sequence $y_{i}, i=1,2, \cdots$, such that for all $i, y_{i}>\epsilon>0$. However

$$
\bar{P}\left(t, y_{i} ; T_{i}, I\right)=\sum_{j=-\infty}^{j-\infty} \int_{j}^{j+1 / 2} \frac{e^{-\left(x-\operatorname{arotan} y_{i} / x+1 / 2\right)^{2} / 2 h_{i}}}{\left(2 \pi h_{i}\right)^{1 / 2}} d x .
$$

For $h_{i}$ sufficiently small, only the term with $j=0$ will contribute appreciably to the sum, as the argument in the first example shows, so

$$
\bar{P}\left(t, y_{i} ; T_{i}, I\right) \doteq \int_{0}^{1 / 2} \frac{e^{-\left(x-\arctan y_{i} / \pi+1 / 2\right)^{2} / 2 h_{i}}}{\left(2 \pi h_{i}\right)^{1 / 2}} d x .
$$

We choose the $y_{i}$ to be $y_{i}=\tan \pi\left(1 / 2-h_{i}\right)$, so for $h_{i} \rightarrow 0, y_{i} \rightarrow \infty$. Then

$$
\bar{P}\left(t, y_{i} ; T_{i}, I\right)=\int_{0}^{1 / 2} \frac{e^{-\left(x+h_{i}\right)^{2} / 2 h_{i}}}{\left(2 \pi h_{i}\right)^{1 / 2}} d x=\int_{h_{i}}^{\left(1 / 2+h_{i}\right) / h_{i}} \frac{e^{-u^{2} / 2}}{(2 \pi)^{1 / 2}} d u .
$$

As $i \rightarrow \infty, h_{i} \rightarrow 0$, so

$$
\lim _{i \rightarrow \infty} \bar{P}\left(t, y_{i} ; T_{i}, I\right)=\int_{0}^{\infty} \frac{e^{-u^{2} / 2}}{(2 \pi)^{1 / 2}} d u=1 / 2 .
$$

Hence $\mathrm{D}(u, u,, u)$ is not satisfied. However $\mathrm{D}(u, u,, b)$ is satisfied, as an argument similar to that in the first example will show. That $\mathrm{C}(u, b, \epsilon, o(h))$ is satisfied may also be seen by a similar argument. We have here an illustration of the distinction made in Theorem VI between the parts (i) and the remainder of the theorem.

We now construct a stochastic process for which almost all sample function have unique right- and left-hand limits, not necessarily finite. We take $\left\{x_{t}(\omega), 0 \leqq t \leqq T^{\prime}\right\}$ to be a Brownian motion process with $x_{0}(\omega)=0, \alpha=1$. For each $\omega$, we define $\tilde{x}_{t}(\omega)$ as follows:

$$
\begin{aligned}
& \tilde{x}_{t}(\omega)=x_{t}(\omega)-4 k, \quad \text { if } 4 k-1 \leqq x_{t}(\omega) \leqq 4 k+1, \\
& \tilde{x}_{t}(\omega)=4 k-2-x_{t}(\omega), \quad \text { if } 4 k+1 \leqq x_{t}(\omega) \leqq 4 k+3, \\
& k=\cdots-1,0,1, \cdots .
\end{aligned}
$$

We suppose $-1 \leqq \gamma<1$. We take

$$
V=\bigcup_{k=-\infty}^{k=\infty}\{x \mid 4 k-1 \leqq x<4 k+\gamma\} \cup\{x \mid 4 k+3-\gamma<x \leqq 4 k+1\} .
$$

Then $\left\{\omega \mid x_{t}(\omega)<\gamma\right\}=\left\{\omega \mid x_{t}(\omega) \in V\right\}$. Hence $\tilde{x}_{t}(\omega)$ is a measurable function so $\left\{\tilde{x}_{t}(\omega), 0 \leqq t \leqq T^{\prime}\right\}$ is a stochastic process. We note that, as before, it can be shown by direct calculation that $P(t, x+4 m ; T, V)=P(t, x ; T, V)$ and $P(t, 4 m+2-x ; T, V)=P(t, x ; T, V)$. Hence, as before, $\widetilde{P}(t, x ; T, I)$ $=P(t, x ; T, V)$. That the $\tilde{x}_{t}(\omega)$ process is a Markov process can be shown by an argument like that used in the previous example. Furthermore, by con- 
struction, $\tilde{x}_{t}(\omega)$ is continuous for almost all $\omega$. We define $y_{t}(\omega)$, for every $\omega$, $t$, as

$$
y_{t}(\omega)=\tan \left[\pi x_{t}(\omega) / 2\right]
$$

In this case, for almost all $\omega, y_{t}(\omega)$ has unique limiting values from the right and from the left (allowing $\infty$ and $-\infty$ as limiting values). Arguments similar to those above will show $\mathrm{C}(u, u, a, o(1)), \mathrm{R}(\infty)$, and $\mathrm{R}(-\infty)$ to be violated, $\mathrm{D}(u, u, u)$ to be satisfied. This process illustrated the distinction made in Theorem VI between the cases (ii), (i), and (iv) or (v).

\section{BIBLIOGRAPHY}

S. Bernstein, ed.

1. Actualités Scientifiques et Industrielles, no. 738, p. 7, Paris, Hermann.

W. DoebLIN

1. Bull. Sci. Math. vol. 62 (1938) pp. 21-32.

2. Skandinavisk Aktuarietidskrift, 1939, pp. 211-222.

3. C. R. Acad. Sci. Paris, no. 207, p. 705.

J. L. DooB

1. Forthcoming book.

2. Trans. Amer. Math. Soc. vol. 42 (1937) pp. 107-140.

3. Ibid. vol. 44 (1938) pp. 87-150.

4. Ibid. vol. 52 (1942) pp. 37-64.

5. Ibid. vol. 58 (1945) pp. $455-473$.

6. Ibid. vol. 63 (1948) pp. 393-421.

W. FELLER

1. Math. Ann. vol. 113 (1936) pp. 113-160.

2. Trans. Amer. Math. Soc. vol. 48 (1940) pp. 488-515.

R. FORTET

K. Iто

1. J. Math. Pures Appl. vol. 22 (1943) pp. 177-243.

1. Memoirs of the American Mathematical Society, no. 4, 1951.

A. KolMOGOROFF

P. LEVY

1. Math. Ann. vol. 104 (1931) pp. 415-458.

1. Processus Stochastiques et Mouvement Brownien, Paris, Gauthier-Villars, 1948.

J. VILLE

2. Pisa Annali (2) vol. 3 (1934) pp. 337-366.

1. Notion de collectif, Paris, Gauthier-Villars, 1939.

N. WIENER

1. Fourier transforms in the complex domain, R. E. A. C. Paley and N. Wiener, Amer. Math. Soc. Colloquium Publications, vol. 19.

UNIVERSITY OF ILLINOIS, URBANA, ILL. 